\title{
Causas y consecuencias de la corrosión de una tubería de acero galvanizado
}

\section{Causes and effects of the corrosion of a plumbing galvanised steel}

\author{
F. J. ALEJANDRE SÁNCIIEZ, R. LUCAS RUIZ
}

Dpto. Construcciones Arquitectónicas II, E.U.A.T. de Sevilla

Fecha de recepción: 16-VI-01

ESPAÑ

\section{RESUMEN}

La durabilidad de los galvanizados en caliente como recubrimientos protectores de la corrosión del acero dependen básicamente de tres factores: la calidad del recubrimiento (espesor). la correcta instalación del elemento galvanizado, y la agresividad del medio al que se encuentren expuestos.

En este trabajo se estudian las causas y consecuencias del deterioro sufrido por una tubería de acero galvanizado, destinada durante unos 30 años a la conducción de agua potable de la red en el mercado del Parque Alcosa (Sevilla). La investigación se ha dividido en tres etapas: en la primera, se ha reali-ado el análisis formal y la caracterización mediante $D R X$ de los productos de alteración generados en el interior del tubo: en la segunda, se han determinado las características físicas del galvanizado según UNE 37-505-89, se ha estudiado la morfología del ataque corrosivo mediante microscopia metalográfica y se ha evaluado la calidad del agua circulante; $y$, por tiltimo, en la tercera, se han cfectuado los cálculos relativos a la disminución real de diámetro, sección y volumen interno, asi como consideraciones sobre la pérdida de carga generadas en la tubería.

Los resultados obtenidos indican que la tubería estaba correctamente instalada y cumplía los requisitos de calidad exigidos, por lo que las causas de su corrosión son atribuibles a la existencia de an medio agresivo para el galianizado.

\section{SUMMARY}

Hot dip galvanising life as a corrosion protective coatings on steel depends basically on three factors: coatings quality (thickness), correct installation of the galvanised article, and weathering effects.

The aim of this research it is to study the decaying causes and effects of a plumbing installation made with hot dip galvanised steel tubes. The mentioned installation was of use during 30 years in the water supply system of the Parque Alcosa market (Sevilla, Spain). The research was divided in three parts: in the first one, shape analysis and XDR of internal decayed products vas carried out; in the second one. physical propierties were determined according to UNE 37 . 505-89, corrosion morphology was conducted by metalographic microscopy, and it was evaluated also water quality; and finally, in the third one, diameter, section, and internal volume decrease was calculated in order to evaluate friction increase and pressure decrease in the installation.

The results obtained show the correct plumbing installation according to quality requirements (UNE 37-509-89), so corrosion causes are mainly attributed to an aggressive environment for the hot dip galvanising. 


\section{INTRODUCCIÓN: EI GALVANIZADO EN CALIENTE}

\subsection{Obtención y estructura}

Con el nombre de galvanizado en caliente se conoce al procedimiento mediante el cual se obtiene un recubrimiento protector de zinc y aleaciones de zinc-hierro sobre piezas o elementos de acero al sumergirlas en un baño de zinc fundido (445-460 'C). A estas temperaturas, el zinc y el acero reaccionan rápidamente formando una serie de cuatro capas consecutivas de aleaciones zinc-hierro: Gamma, Delta, Zeta y Eta, nombradas desde el interior al exterior, con distintos espesores y contenidos en hierro decrecientes $(25 \%, 10 \%, 6 \%$ y $» 0 \%$, respectivamente) (Notas Informativas de la ATEG).

Los espesores medios conseguidos con el galvanizado en caliente dependen de las técnicas que se apliquen para su control (centrifugado, soplado, etc.), y suelen estar comprendidos entre las $20 \mu \mathrm{m}$ y $100 \mu \mathrm{m}$. Este intervalo equivale al de 144 y $720 \mathrm{gr} / \mathrm{m}^{2}$ si se expresa en masas medias de recubrimiento por unidad de superficie, y tomando como densidad del recubrimiento el valor de $7,2 \mathrm{gr} / \mathrm{cm}^{3}$ (UNE 37-505-89 y UNE-EN-ISO 1461).

\subsection{Protección electroquímica}

Los recubrimientos galvanizados además de proporcionar una gran dureza y resistencia a la abrasión (sobre todo en las capas más internas), se caracterizan, ante todo, por proporcionar al acero de base la denominada protección electroquímica catódica. Este tipo de protección se basa en las reacciones que se producen cuando se unen dos metales de distinta actividad electroquímica, y, por tanto, con distinto potencial eléctrico, así el metal más activo ( $\mathrm{Zn}$, en este caso) sufre la ionización: $\mathrm{Zn} \rightarrow \mathrm{Zn}^{+2}+2 \mathrm{e}$ o reacción anódica, cediendo electrones al más noble (Fe, en este caso), de forma que se disminuye $\mathrm{c}$ incluso se impide su ionización. En la Figura 1 se muestran los procesos que tienen lugar en un medio acuoso:

Como se puede observar en la Figura 1, la capa de zinc sobre el acero impide su oxidación al sacrificarse éste para protegerlo. Esta protección electroquímica durará mientras quede algo de zinc sobre el accro, y su alcance scrá mayor mientras mayor sea la conductividad del medio'. Por este motivo, y como diferencia importante con la protección antioxidante que ofrecen las pinturas. si se produce una pequeña discontinuidad en el galvanizado quedando al descubierto el acero de base, éste no se oxidará mientras quede zinc en los alrededores.

Este comportamiento también justifica que se pueda emplear indistintamente para especificar los recubrimientos galvanizados: el espesor (en micras) o la masa de recubrimiento por unidad de superficie $\left(\mathrm{gr} / \mathrm{m}^{2}\right)$, ya que la continuidad del mismo no es un requisito esencial.

\subsection{Aplicaciones}

Son innumerables los elementos de hicrro y acero que son objeto del proceso de galvanización para protegerlos frente a la oxidación. Algunos de los ejemplos más habituales son: tubos, chapas, alambres, perfiles, tornillos, armaduras para el hormigón, etc.

\subsection{Características y propiedades de los galvanizados}

Según las normas UNE 37-505-89 y la UNE-EN-ISO1999, para los recubrimientos galvanizados en caliente sobre tubos de acero se establecen las características químicas y las físicas (Tabla 1 ).
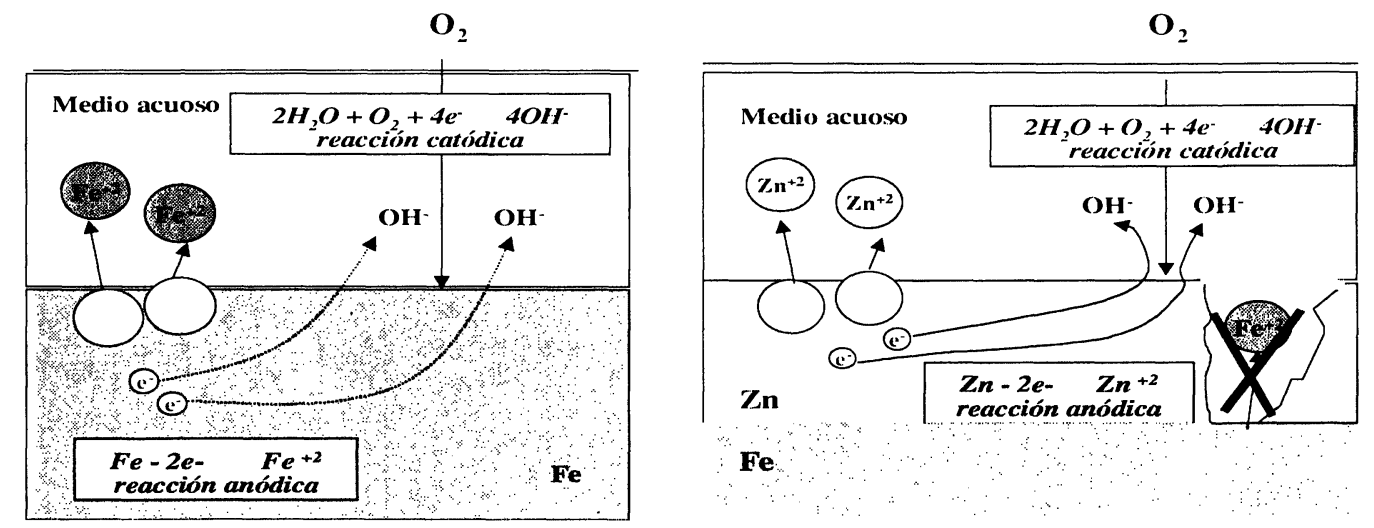

Figura 1.- Izda. Reacciones de corrosión de una pieza de hierro en un medio acuoso aireado. Deha. Protección electroquímica catódica en la que el zinc se sacrifica para proteger al hierro

\footnotetext{
'Se ha de tener en cuenta que para cerrar las reacciones catódicas y anódicas es necesario que el medio sea conductor. Segén II. H. Uhlig ( 195.5 ). en agua de mar el cinc puede proteger al acero expuesta a una distancia de varias pulgadas. mientras que en agua pura. peyueñas áreas expuestas, se corroen en poco tiempo.
} 
TABLA 1

Características físicas y químicas de los galvanizados según norma UNE

\begin{tabular}{||l|l|c|c|c||}
\hline & CARACT. & \multicolumn{3}{|c||}{ CARACTERÍSTICAS FÍSICAS } \\
\cline { 3 - 5 } & QUÍMICAS & Aspecto & Espesor $(\mathrm{min})$. & Adherencia \\
\hline \multirow{3}{*}{ UNE 37-505- } & Zn $>98 \%$ & Liso, sin & & Función del $\varnothing$ \\
1989 & Impurezas $<2 \%$ & discontinuidades y & $>400 \mathrm{gr} / \mathrm{m}^{2}$ & $\varnothing<50 \mathrm{~mm}$ \\
& exento de manchas & $(\approx 55 \mu \mathrm{m})$ & ensayo de doblado \\
\hline \multirow{3}{*}{ UNE-EN- } & Zn $>98,5 \%$ & Sin ampollas, & Función del espesor de acero & \\
ISO-1999 & Impurezas & rugosidades y zonas & $\geq 1,5 \mathrm{~mm}$ hasta $<3 \mathrm{~mm}$ & No es necesario \\
& (excepto Fe, $\mathrm{Sn})$ & no recubiertas. Exento & $325 \mathrm{~g} / \mathrm{m}^{2}(45 \mu \mathrm{m})$ Local & \\
& $<1.5 \%$ & de residuos, grumos... & $395 \mathrm{~g} / \mathrm{m}^{2}(55 \mu \mathrm{m})$ Medio & \\
\hline
\end{tabular}

\subsection{Durabilidad}

La duración en servicio de los galvanizados va a depender, principalmente, de tres parámetros: la calidad del recubrimiento (sobre todo su espesor), la correcta ejecución, y la agresividad de los medios a los que se encuentren expuestos.

Para la exposición atmosférica la norma ISO 9223-1992, cstablece distintas categorías de corrosividad que generan diferentes velocidades de corrosión del zinc dependiendo de:

1. Nivel de humedad ambiental. La presencia de agua es vital para estabilizar los iones formados en las reacciones electroquímicas, además, su conductividad permite el cierre del circuito eléctrico al facilitar el desplazamiento de las especies formadas.

2. Contenido de contaminantes en el aire. La presencia de ácidos (lluvia ácida) es muy perjudicial para el zinc ya que éste es atacado por medios con $\mathrm{pH}$ inferior a 6. También los cloruros son muy dañinos porque pueden romper localmente las películas pasivas originando fenómenos de corrrosión por picadura.

En el caso más concreto de las instalaciones de fontanería realizadas con tubo de acero galvanizado, las condiciones de trabajo en sus superficies internas y externas

son diferentes, teniéndose que distinguir entre la corrosión externa e interna (UNE 37-509-89):

\section{Zona externa}

1. Presencia de humedad. Se debe evitar por los motivos citados anteriormente. Si existen materiales agresivos éstos actuarán normalmente en presencia de agua.
2. Utilización de yeso o escayola. El pH de este material es cercano a 6, y en este medio ácido el zinc comienza a disolverse como $\mathrm{Zn}^{+2}$ en las regiones anódicas. El anión $\mathrm{SO}_{4}{ }^{\prime \prime}$ también es perjudicial porque puede romper localmente las películas pasivas, originando fenómenos de corrosión por picadura.

3. Cubrición directa de las tuberías con materiales heterogéneos que generan discontinuidades en la superficie, originando pilas por aireación diferencial ${ }^{3}$.

4. Ejecución incorrecta. La estructura idónea del recubrimiento exterior de la tubería es la formada por lechada de cemento, y después por un mortero rico en cemento ( $1-2$ $\mathrm{cm})$.

\section{Zona interna}

1. Características del agua. Son varios los parámetros del agua que afectan a la durabilidad:

1.1. Temperatura. En el caso del zinc, cuando las temperaturas del agua son superiores a los $60^{\circ} \mathrm{C}$, los productos de corrosión pasan de $\mathrm{Zn}(\mathrm{OH})$, a $\mathrm{ZnO}$, pudiendo dar lugar a la inversión de la polaridad del zinc respecto a la del hierro, lo que genera el ataque por picadura del acero del tubo. La temperatura de inversión está influida por la concentración de diversos tipos de sales (H.H. Uhlig ,1955; Otero, 1997).

1.2. Acidez. Su influencia se ha comentado anteriormente.

1.3. Contenido de sustancias agresivas. Además de la presencia de sales perjudiciales disueltas $\left(\mathrm{SO}_{4}{ }^{-} \mathrm{y} \mathrm{Cl}{ }^{-}\right)$, el agua puede llevar disueltos gases como el $\mathrm{O}_{2}$ y el $\mathrm{CO}_{\text {. }}$ El oxígeno es necesario para que se dé la reacción catódica, por tanto, su ausencia evita la corrosión. Cuando se encuentra de forma heterogénea (burbujas de aire, zonas de sedimentos) favorece la corrosión por aireación diferencia.

\footnotetext{
Se muestran los espesores y massas de recubrimiento para un intervalo de espesor de acero $>1.5 \mathrm{~mm}$ hasta $<3 \mathrm{~mm}$, ya que para el tubo de acero 1.5 » de diámetro el espesor suele ser de $2.6 \mathrm{~mm}-2.8 \mathrm{~mm}$.

'La aireación diferencial es un tipo de hetterogeneidad del medio que se produce cuando algunas partes de la superficie quedan eon menos disponibilidad de $\mathrm{O}_{2}$ que otras. En la zona mal aireada se llega a consumir el $\mathrm{O}_{\text {. }}$ y pasa a actuar como región anodica. mientras que las zonas mejor aireadas se convierten en zonas catódicas.
} 
La presencia de $\mathrm{CO}_{2}$ puede cambiar los productos de corrosión. p.ej. de hidróxidos a carbonatos básicos con menor poder protector favoreciendo la corrosión del zinc. También interviene en el equilibrio $\mathrm{CO}_{3}{ }^{-} / \mathrm{HCO}_{3}$ desplazándolo hacia el segundo. que es parcialmente soluble, frente a los carbonatos, que son insolubles y que polarizan la reacción catódica disminuyendo la velocidad de corrosión (Otero, 1997).

1.4. Índice de saturación de Langelier. Es un concepto que indica cual es la capacidad de precipitación de capas calcáreas protectoras, se define como:

siendo,

$$
\mathrm{IS}=\mathrm{pH}_{\text {re:al }}-\mathrm{pHs}
$$

$\mathrm{pH}_{\text {real }}=\mathrm{pH}$ medido en el agua, y $\mathrm{pH}=\mathrm{pCa}+\mathrm{pAlc}+$

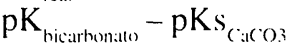

Si el IS es positivo, indica que el agua está sobresaturada en $\mathrm{CaCO}_{3}$ y tendrá tendencia a la formación de capas protectoras. Si es negativo, el $\mathrm{CaCO}_{3}$ tenderá a disolverse formando bicarbonatos y el agua será agresiva.

2. Entrada de aire en la instalación. Su influencia se ha comentado en el apdo. 1.3.

3. Entrada de materiales sólidos. Generan discontinuida-

des en la superficie que dan lugar a la formación de pilas por aireación diferencial (apdo. 1.3.).

4. Mala ejecución. Son múltiples los fallos de ejecución que pueden acelerar la corrosión: el contacto entre metales distintos que genera nuevos pares galvánicos $(\mathrm{Cu}-\mathrm{Fe})$, el empleo de soldadura para unión, aplicación de calor para el curvado destruye el galvanizado, etc.

\section{ESTUDIOS EXPERIMENTALES}

Partiendo de 3 muestras de unos $50 \mathrm{~cm}$ de longitud de una tubería de acero galvanizado de 1,5 " de $\phi$ se ha efectuado la caracterización de los productos de la corrosión, el estudio de la morfología de la corrosión, la determinación de las características físicas del galvanizado no oxidado, la evaluación de las características del agua circulante, y, por último, cálculos relativos a la disminución de sección y de diámetro sufridos en la tubería.
2.1. Caracterización de los productos resultantes de la corrosión

Para poder examinar la morfología de los productos formados en el interior del tubo, se procedió a su llenado con resinas para evitar su rotura cuando se realizaran los cortes transversales y longitudinales.

En la Figura 2 se observa que en la parte interna del tubo se han gencrado unas formaciones muy voluminosas, porosas, de baja de densidad y con un espesor poco uniforme. A priori se pueden atribuir a productos derivados de la corrosión del acero (oxi-hidróxidos de hierro) aunque no se descarta la posible presencia de sales precipitadas del agua.

\subsubsection{Caracterización mineralógica mediante DRX}

Se ha llevado a cabo sobre una muestra obtenida mediante el raspado de los productos existentes en el interior tubo.

En la Figura 3 se muestra el difractograma correspondiente para la identificación de las fases cristalinas, que se han realizado con el software Diffrac-at Siemens 1991 package.

Los resultados muestran cómo todos los productos formados en el interior del tubo provienen de la corrosión del acero al ser todos oxi-hidróxidos de hierro. No se han detectado ni sales procedentes del agua (carbonatos, cloruros, sulfatos, etc.), ni compuestos procedentes del $\mathrm{Zn}$.

\subsection{Morfología de la corrosión}

Para realizar este estudio se pulimentaron las secciones transversales, posteriormente se trataron con Nital, y, finalmente. sc observaron con un microscopio metalográfico (50X). Figura 4 ..

Del análisis de las tres fotografías se puede extraer la siguiente información:

- El acero de la tubería está constituido por perlita en una matriz de ferrita.
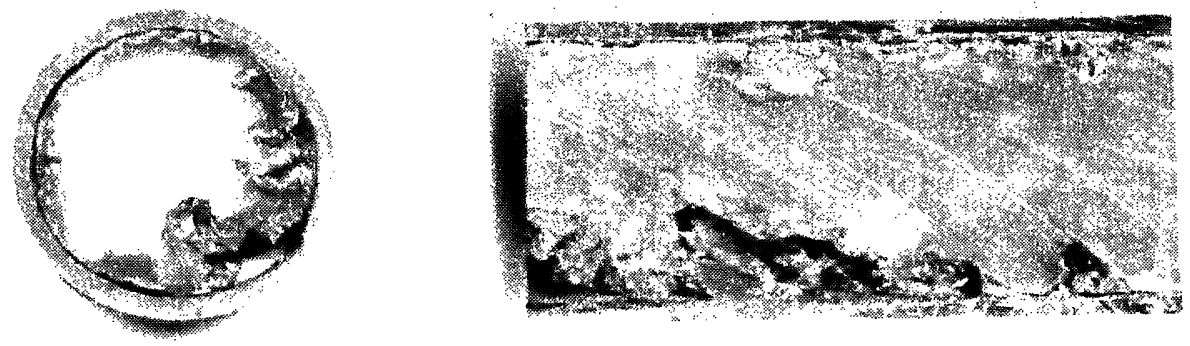

Figura 2.- Corte transiersal ! longitudinal del tuho de acero galsanizado. Se puede ohservar lat presencia de productos voluminosos con un espesor mus imegular atribuibles a oxidos de hierro. 


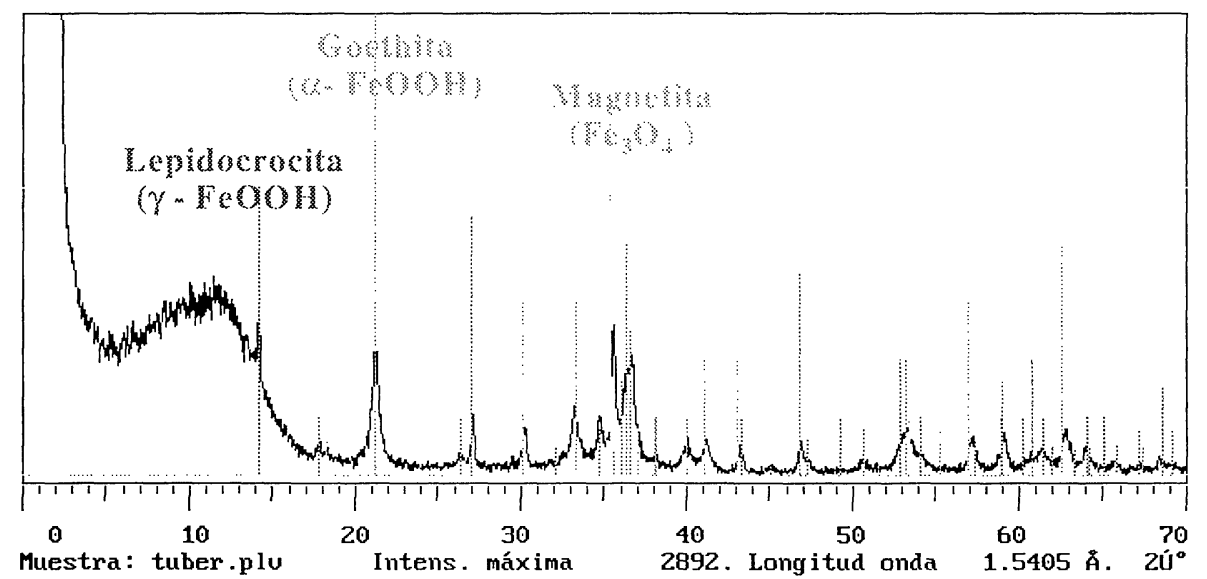

Figura 3.- Difractograma de RX (método de polvo) en el que se identifican los diferentes óxidos de hierro, resultado de la corrosión del acero galvanizado.

- La capa de galvanizado exterior se conserva, por lo general, en buenas condiciones (foto 2), aunque en determinadas zonas puntuales ha desaparecido (foto 3). El galvanizado interior ha desaparecido por completo.

Existen distintos tipos de corrosión de acuerdo con la morfología del ataque:

a) Corrosión uniforme. Se puede observar en la superficie de la Foto 1, en la que el ataque se extiende de forma homogénea sobre toda la superficie metálica.

b) Corrosión intergranular. En las Fotos 1 y 2 se puede apreciar la existencia de corrosión en los límites de grano del material metálico. Este tipo de corrosión tiene su fundamento en las heterogeneidades e irregularidades que presentan los límites de grano.

1

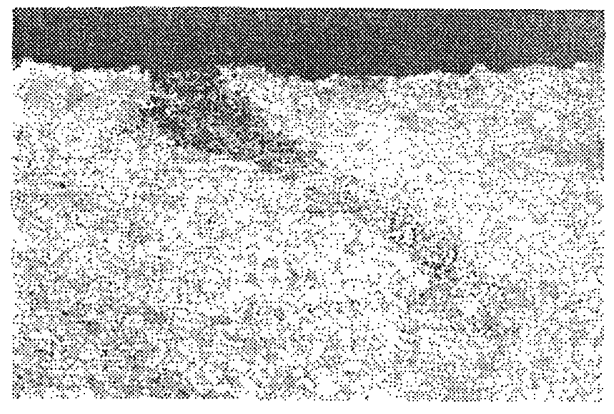

3

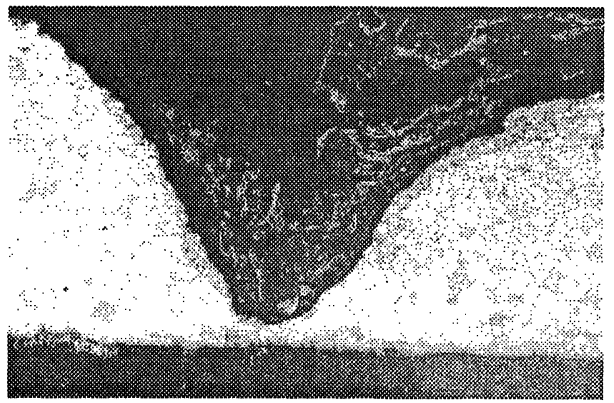

c) Corrosión por picadura. Este tipo suele producirse cuando existen heterogeneidades superficiales como la rotura local de la capa de galvanizado. Así, el ataque comienza en zonas aisladas con la formación de pequeños túneles (Foto 1) que avanzan hacia el interior con rapidez debido a que la velocidad de corrosión suele ser elevada (Foto 2).

Finalmente, debido a la formación de capas porosas de $\mathrm{Fe}(\mathrm{OH})$, que cubren la salida de la picadura (Foto 3), se deja parcialmente aislado el interior de la misma, originando la acidificación local como consecuencia de la hidrólisis de parte del catión $\mathrm{Fe}^{+2}$ según la reacción:

$\mathrm{Fe}^{+2}+\mathrm{H}_{2} \mathrm{O}-\mathrm{Fe}(\mathrm{OH})^{+}+\mathrm{H}^{+}$, en estas condiciones el ataque al acero es más intenso.

2

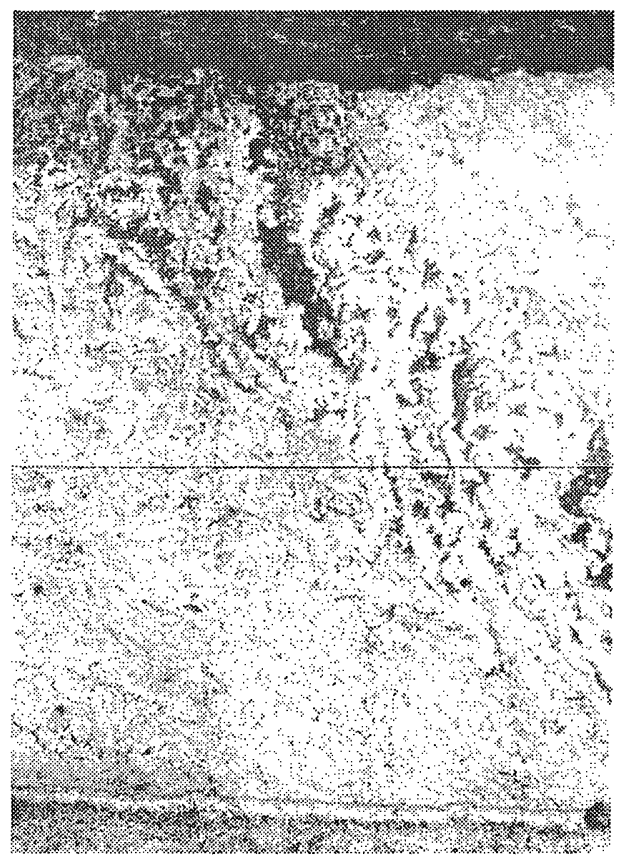

Figura 4.- Fotografías al microscopio metalográfico $(50 \mathrm{X})$ de varias secciones transversales 


\subsection{Evaluación de las características del agua circu- lante}

Para conocer la capacidad de precipitación de carbonatos del agua potable distribuida en red durante los últimos años, se solicitó a Emasesa ${ }^{4}$ información sobre el índice de saturación, suministrando los resultados expuestos en la Tabla 2.

Se puede apreciar cómo el signo que presenta el indice de saturación del agua de red durante el período 19982000 es negativo, lo cual se traduce en una nula capacidad de formación de capas protectoras de "cal". Este hecho confirma que no se encontraran carbonatos entre los productos resultantes del deterioro de la tubería.

\subsection{Características físicas del galvanizado no oxida- do}

En las muestras estudiadas el galvanizado interior había desaparecido por completo, en cambio, el exterior se encontraba sin oxidar por lo que se determinaron sus propiedades físicas según UNE 37-505-89, Tabla 3.

Se puede afirmar que el galvanizado exterior cumple sobradamente los espesores exigidos para esta aplicación, su estado de conservación ha sido bueno ya que a pesar de encontrarse enterrado estaba recubierto en su totalidad por mortero rico en cemento.

Teniendo en cuenta la técnica de inmersión de la galvanización en caliente, se puede deducir que el recubrimiento interior también debía de cumplir los espesores mínimos recomendados.

\subsection{Cálculos de la disminución de sección y de diámetro sufridos por la tubería}

Con las 3 muestras de tubería saturadas en agua durante 24 horas y mediante pesada hidrostática se llevó a cabo la detcrminación de la disminución de volumen $(\% \Delta \mathrm{V})$ en el interior de los mismos, la sección interior equivalente (Si eqv), y el diámetro interior equivalente ( $\phi i$ eqv), Tabla 4.

Se puede observar cómo la disminución de sección y de volumen interior medio ha sido bastante elevada $(21,9 \%$ y $22,1 \%$, respectivamente), casi $1 / 3$ en algunas zonas:

La disminución del diámetro interno de la tubería, unida al aumento de la velocidad de circulación del agua, y al aumento del coeficiente de fricción producido por el mayor rozamiento existente entre el agua y los voluminosos

TABLA 2

IS del agua de red

\begin{tabular}{||l|c|}
\hline AÑ & IS \\
\hline 1998 & $-0,74$ \\
\hline 1999 & $-0,71$ \\
\hline 2000 & $-0,66$ \\
\hline
\end{tabular}

TABLA 3

Algunas propiedades de los restos de galvanizado

\begin{tabular}{||c|c|c|c||}
\hline \hline Prop. & Aspecto & Espesor & Adherencia \\
\cline { 2 - 4 } Físicas & $\begin{array}{c}\text { Gris metálico mate con restos de mortero de cemento/ } \\
\text { Picaduras localizadas procedentes del interior }\end{array}$ & $\begin{array}{c}653 \mathrm{gr} / \mathrm{m}^{2} \\
(\approx 90 \mu \mathrm{m})\end{array}$ & $\begin{array}{c}\text { No realizado por insuficiente } \\
\text { tamaño de muestra }\end{array}$ \\
\hline
\end{tabular}

TABLA 4

Calculos de la disminución de sección y de diámetro sufridos por la tubería

\begin{tabular}{|c|c|c|c|c|c|}
\hline M UESTRA S & $\% \Delta \mathrm{V}_{\text {int }}$ & $S_{\text {iegv }}\left(\mathrm{cm}^{2}\right)$ & $\% \Delta \mathrm{S}_{\text {int }}$ & $\varnothing_{\text {ieqv }}(\mathrm{cm})$ & $\% \Delta \varnothing_{\text {int }}$ \\
\hline Tubo 1 & $-2,8,4$ & 7,3 & $-28,4$ & 3,0 & $-16,6$ \\
\hline Tubo 2 & $-7,8$ & 9,4 & $-7,8$ & 3,4 & $-5,5$ \\
\hline Tubo 3 & $-18,6$ & 8,3 & $-18,6$ & 3,2 & $-11,1$ \\
\hline Media & $-18,2$ & 8,3 & $-18,2$ & 3,2 & $-11,0$ \\
\hline Tubo 1,5" nuevo & 0 & 10,2 & 0 & 3,6 & 0 \\
\hline
\end{tabular}

${ }^{4}$ Empresa Municipal de Abastecimiento y Sancamiento de Aguas de Sevilla, S. A.

${ }^{5}$ Los datos de espesor se comprobaron también mediante la observación con microscopio metalográfico de varias de las seceiones 8transversales y longitudinales), dando un espesor aproximado de 80 micras. 
e irregulares óxidos de hierro generados (Fórmula de Nikuradse de tubos rugosos y régimen turbulento) (Figura 5), origina según la fórmula general de Darcy-Weisbach una importante caída de carga (Mayol, 1997) y la consiguiente disminución del caudal suministrado por la tubería:

\section{Fórmula de Nikuradse}

Fórmula general de Darcy-Weisbach

$$
\frac{1}{\sqrt{\lambda}}=2 \log \left(\frac{3,71 \mathrm{D}}{\mathrm{K}}\right) \quad \mathrm{J}=\lambda \frac{\mathrm{V}^{2}}{2 \mathrm{gD}} \mathrm{L}
$$

siendo:

$\lambda$, coeficiente de fricción

$\mathrm{D}$, diámetro interior

$\mathrm{K}$, rugosidad de la tubería

$\mathrm{J}$, pérdida de carga en la tubería

$\mathrm{G}$, aceleración de la gravedad

$\mathrm{V}$, velocidad de circulación del agua

L, longitud de la tubería

\section{CONCLUSIONES}

A pesar de la naturaleza compleja del fenómeno de la corrosión y de los múltiples factores que suelen intervenir en su origen, se pueden concluir de este estudio los siguientes puntos:

- Las características ensayadas en los restos de galvanizado existentes parecen indicar la alta calidad del recubrimiento inicial de zinc sobre la tubería.

- La corrosión se ha originado en el interior de la tubería, alcanzando una grản extensión y profundidad, y llegando a generar picaduras que han perforado el espesor de la misma.

- La corrosión en el interior de la tubería no se ha producido de forma acelerada, ya que período de servicio ha sido amplio, de unos 30 años. En su origen posiblemente han intervenido factores desarrolladores de pilas de aireación diferencial como la entrada de aire y sólidos (incluidos los óxidos formados), que combinados con la incapacidad del agua de red circulante de formar capas calcáreas protectoras han adquirido una significación importante.
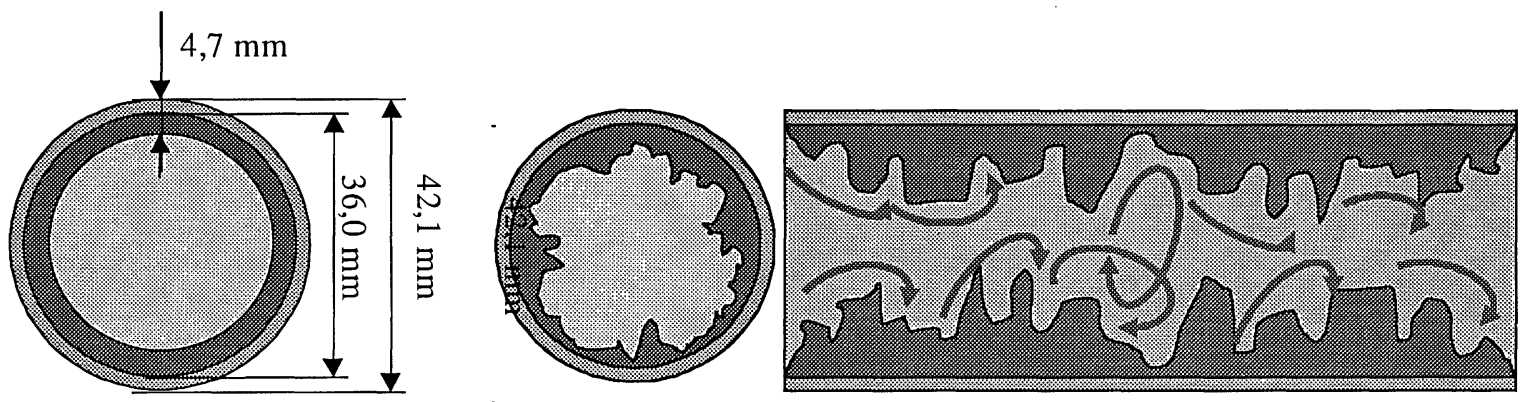

Figura 5.- Izda. Sección mostrando los diámetros equivalentes. Ctro. y dcha. Secciones transversales y longitudinales mostrando el régimen turbulento.

\section{BIBLIOGRAFÍA}

(1) AENOR. Norma UNE 37-501-88. Galvanización en caliente. Características y métodos de ensayo, 1988.

(2) AENOR. Norma UNE 37-505-89. Recubrimientos galvanizados en caliente sobre tubos de acero. Características y métodos de ensayo, 1989.

(3) AENOR. Norma UNE 37-509-89. Instalaciones de fontanería realizadas con tubo de acero galvanizado. Recomendaciones para la prevención de la corrosión prematura, 1989.

(4) AENOR. Norma UNE-EN-ISO 1461. Recubrimientos galvanizados en caliente sobre productos acabados de hierro y acero. Especificaciones y métodos de ensayo, 1999.

(5) Asociación Técnica Española de Galvanización. Galvanización en caliente, notas informativas. Madrid.

(6) E. Otero Huerta. Corrosión y degradación de materiales. p. 365, Editorial Síntesis S.A., Madrid, 1997.

(7) H. H. Uhlig. The corrosion Handbook. pp. 1163, John Wiley \& Sons, Inc., Ncw York, 1955.

(8) J. M. Mayol Mallorquí. Tuberías, tomo 1 (materiales, cálculos hidráulicos, cálculos mecánicos). Librería Editorial Bellisco, Madrid, 1997. 